\title{
Rifaximin for Irritable Bowel Syndrome (IBS) in Gulf War Veterans: Losing the Battle but Winning the War?
}

\author{
Lucinda A. Harris ${ }^{1}$ \\ Published online: 6 February 2019 \\ (c) Springer Science+Business Media, LLC, part of Springer Nature 2019
}

Conquering the treatment of irritable bowel syndrome (IBS) is somewhat like trying to win a war. Many enemies have been implicated in its causation, among them visceral hypersensitivity, alterations in intestinal permeability and motility, brain-gut dysregulation, defects in the autonomic nervous system, alterations in GI immune function, hormones, psychosocial factors, and most recently alterations in the gut microbiome. Many have realized that the symptoms of small intestinal bacterial overgrowth (SIBO) such as changes in bowel habit, cramping, and bloating to mention just a few are remarkably similar to IBS. Therefore, testing for SIBO and treating it with antibiotics, particularly the poorly absorbable broad-spectrum antibiotic rifaximin, have conceptually evolved in the area of IBS therapies [1]. Further support for the post-infectious nature of IBS is also found in the identification of biomarkers that are antibodies to bacterial toxins in patients who have IBS with diarrhea (IBSD) or mixed bowel habits (IBS-M) [2] combined with the observation that chronic diseases such as IBS can often follow infectious gastroenteritis [3]. With over $50 \%$ of military personnel having gastroenteritis while being deployed in the Gulf, this would seem to be an ideal population in which to study post-infectious IBS. In this issue of Digestive Diseases and Sciences, Tuteja et al. reported that up to a third of Gulf War (GW) Veterans have IBS, and despite the pressures of war and posttraumatic stress disorder, the biggest risk factor for getting IBS may indeed be prior infection [4]. Given the high number of subjects identified, the authors tested the hypothesis that rifaximin would "normalize SIBO" and thus reduce IBS symptoms.

In this double-blind, placebo-controlled trial, the authors identified 120 GW Veterans meeting Rome III criteria for

Lucinda A. Harris

Harris.Lucinda@mayo.edu

1 Division of Gastroenterology and Hepatology, Mayo Clinic College of Medicine, 13400 E. Shea Blvd, Scottsdale, AZ 85259, USA
IBS [4]. Fifty of those with non-constipated IBS were randomized after lactulose hydrogen breath testing (LHBT) to receive either rifaximin $550 \mathrm{mg}$ or placebo b.i.d. after a 2-week "run-in" period. Patients were advised to not change medications or diet during the study period. Evaluated outcome measures included stool frequency, stool consistency (via the Bristol stool scale), urgency, severity of abdominal pain, severity of bloating, and global improvement. Quality of life (QoL) was assessed with the IBS-QoL scale. Lactulose breath testing was performed at baseline and again after 2 weeks of treatment. Only 44 patients completed the study (38 men, 6 women, median age $=52$ ). Rifaximin was not associated with significant improvement in global symptoms, abdominal pain, stool frequency, urgency, bloating, or stool consistency (all $P \geq 0.25$ ) or QoL (all $P \geq 0.26$ ). Lactulose breath testing showed no difference between rifaximin and placebo ( $7 \%$ vs. $22 \%, P=0.54$ ).

For those who believe IBS is post-infectious disorder, this result would seem to be disappointing, but before taking that stance, one has to thoughtfully evaluate the study. One of the strengths of the study is that the patients were properly randomized and patients and clinicians were blinded as to the treatment. Another strength was that they used proper diagnostic criteria including ensuring that the patients met Rome III stool criteria and that symptoms had been present for over 6 months. They also use validated questionnaires to assess individual symptoms, global improvement, and quality of life.

Despite these strengths, weaknesses were also present. The sample size is very small: out of the 120 patients screened, only 50 met all the inclusion/exclusion criteria. Moreover, six patients withdrew from the study, leaving only 44 subjects to be randomized. Instead of the hoped for $N=64$, which would have provided $80 \%$ power to detect a standard deviation of 0.5 , the $N=44$ left them with an $80 \%$ power to detect a SD of 0.86 . Although the authors maintain that they had sufficient numbers, statistically one could equally argue that the number of subjects was indeed too small. 
This study was also unusual for IBS studies in having a predominantly male population. In past rifaximin trials, particularly those upon which approval was garnered, the study population was predominantly female $(\geq 70 \%)$, whereas this study population was $86 \%$ male [5]. Certainly, it makes sense that there are more men because the armed forces are predominantly male, but the literature supports IBS as being a femalepredominant disorder. The authors by their own admission discount psychological distress as a confounding factor in this study. They describe that Veterans had milder symptoms of psychological stress on a validated questionnaire and did not find a statistically significant difference in psychological distress between placebo and treatment groups. So why were so many male patients affected with IBS? Are there different microbial factors or biomarkers present in GW Veterans, especially if this population seem less responsive to this treatment?

Further confounding the data is the fact that instead of the FDA-approved dose of $550 \mathrm{mg}$ three times daily, the medication was only given twice daily. The authors rationalize this decision based on the fact that other trials of rifaximin have used twice daily dosing [5] and even point out that a recent study using the higher dose of rifaximin did not show positive results [6], although the latter study was an even smaller mechanistic observational analysis of 24 female patients designed to measure intestinal transit and permeability as well as symptomatic improvement, and was not a clinical trial [7]. One might argue that the Tuteja trial was even less adequately designed to assess symptomatic improvement under the assumption that since the approved rifaximin dose for IBS treatment is $550 \mathrm{mg}$ t.i.d., that dose would have been used in a well-designed trial.

Lastly, the LHBT was used as a marker for bacterial overgrowth. The paper did for the most part follows recent consensus guidelines for performance of the breath test (adequate fasting, avoidance of carbohydrates, proper dose of lactulose, duration of study, and rise in breath hydrogen of $\geq 20 \mathrm{ppm}$ ) [8]. They alternatively used a double peak rise of $\geq 12 \mathrm{ppm}$ as an endpoint even though consensus guidelines have stated that the double peak criterion is invalid. It is not clear from the results which of the two endpoints for breath testing was met for individual subjects. It is also not delineated whether patients were advised against smoking or exercise, two other possible confounders. Approximately $60 \%$ of the patients in both the placebo and treatment group had positive breath tests, a percentage that did not change posttreatment. As the authors point out, lactulose breath testing for diagnosing SIBO and for predicting the response to treatment remains controversial.

Breath methane as well as hydrogen was measured in the study, in accordance with consensus guidelines. Nonetheless, Tuteja, et al. designated a methane producer as having a rise in methane of $>1 \mathrm{ppm}$. Using this definition, approximately $30 \%$ of the patients in the placebo and treatments had positive methane levels that also did not change posttreatment. Since consensus guidelines define a rise of $\geq 10 \mathrm{ppm}$ as a methane producer, the level used by the authors probably falsely inflated the percentage of methane producers and further was not predictive of response to treatment. Since methane is a marker of constipation and hence slow transit, and since these were non-constipated IBS patients, the rationale behind using this low cutoff is not readily apparent.

The authors are to be congratulated for doing one of the first studies of rifaximin in Gulf War Veterans with IBS. It is also a reminder of yet another adverse effect of combat that can occur in our valiant returning Veterans. Though this is a negative study, it challenges investigators to design clinical studies more carefully so as to avoid methodological flaws and thus devise superior research studies. This study also does not negate the observation that infection and SIBO may indeed contribute to IBS pathophysiology and may be useful treatment targets. Despite its flaws, this study should be a reminder that the understanding of the microbiome is indeed just beginning and that Gulf War Veterans may indeed need unique biomarkers for identifying responders to antibiotic therapy and may benefit from the development of novel therapeutic approaches.

\section{References}

1. Sachev AH, Pimentel M. Antibiotics for irritable bowel syndrome: rationale and current evidence. Curr Gastroenterol Rep. 2012;14:439-445.

2. Pimentel M, Morales W, Rezaie A, et al. Development and validation of a bio-marker for diarrhea-predominant irritable bowel syndrome in human subjects. PLOS ONE. 2015;10:e0126438.

3. Porter CK, Choi D, Cash B, et al. Pathogen-specific risk of chronic gastrointestinal disorders following bacterial causes of food-borne illness. BMC Gastroenterol. 2013;8:13-46.

4. Tuteja A, Talley N, Stoddard GJ, et al. Double-blind, placebo controlled study of rifaximin and lactulose hydrogen breath test in Gulf War Veterans with irritable bowel syndrome. Dig Dis Sci. (Epub ahead of print). https://doi.org/10.1007/s1062 0-018-5344-5.

5. Schoenfeld P, Pimentel M, Chang L, et al. Safety and tolerability of rifaximin for the treatment of irritable bowel syndrome without constipation: a pooled analysis of randomized, double-blind, placebo controlled trials. Aliment Pharmacol Ther. 2014;39:1161-1168.

6. Acosta A, Camilleri M, Shin A, et al. Effects of rifaximin on transit, permeability, fecal microbiome, and organic acid excretion in irritable bowel syndrome. Clin Transl Gastroenterol. 2016;26:e173. https://doi.org/10.1038/ctg.2016.32.

7. Correspondence with Dr. Michael Camilleri to determine patients' gender in this study. 1/15/2019.

8. Rezaie A, Buresi M, Lembo A, et al. Hydrogen and methane-based breath testing in gastrointestinal disorders: the North American consensus guidelines. Am J Gastroenterol. 2017;112:775-781.

Publisher's Note Springer Nature remains neutral with regard to jurisdictional claims in published maps and institutional affiliations. 